\title{
A new kind of scientist
}

\author{
GAVIN SCHMIDT AND ELISABETH MOYER
}

\author{
Climate researchers must begin to bridge disciplinary divides — and institutions must begin to \\ reward them for it.
}

$\mathrm{t}$ is a truth universally acknowledged that interdisciplinary science is essential for furthering understanding of climate change. Two important research efforts spanning several disciplines exemplify this point: first, using past records of climate change to add credibility to projections of future climates, and second, combining climate projections with economics and sociology to evaluate the impacts of change and the potential policies to minimize these impacts. In both cases, preliminary work is proceeding, but too slowly to satisfy the needs of planners and policymakers. It is not enough to intermittently bring researchers in different fields together; to accelerate progress, we need to cultivate a new kind of scientist, one who will be able to more easily bridge these interdisciplinary divides.

But such a challenge is not without its problems, among them the difficulties in penetrating the jargon of another field, mismatches in the problems that are considered important, and differing assumptions about the desired results. Institutional barriers also hinder collaboration. Tenure committees often do not appreciate publications in out-of-discipline journals and may not value collaborative work. Timescales for promotion and metrics of success may be incompatible in different fields. Moreover, inflexible funding sources can stifle innovative approaches.

The two examples mentioned above serve as useful illustrations of the problems in practice. First, climate modelling and palaeoclimatology are fields that nominally focus on the same topic but with very different expectations of data coverage, temporal and spatial scales, and reliance on underlying physics. Climate modellers produce global maps of physical parameters on regularly spaced grids. Palaeoclimatologists are typically constrained to consider impacts and changes over time at only a few locations: for instance, using drilled ocean sediments or glacial ice cores

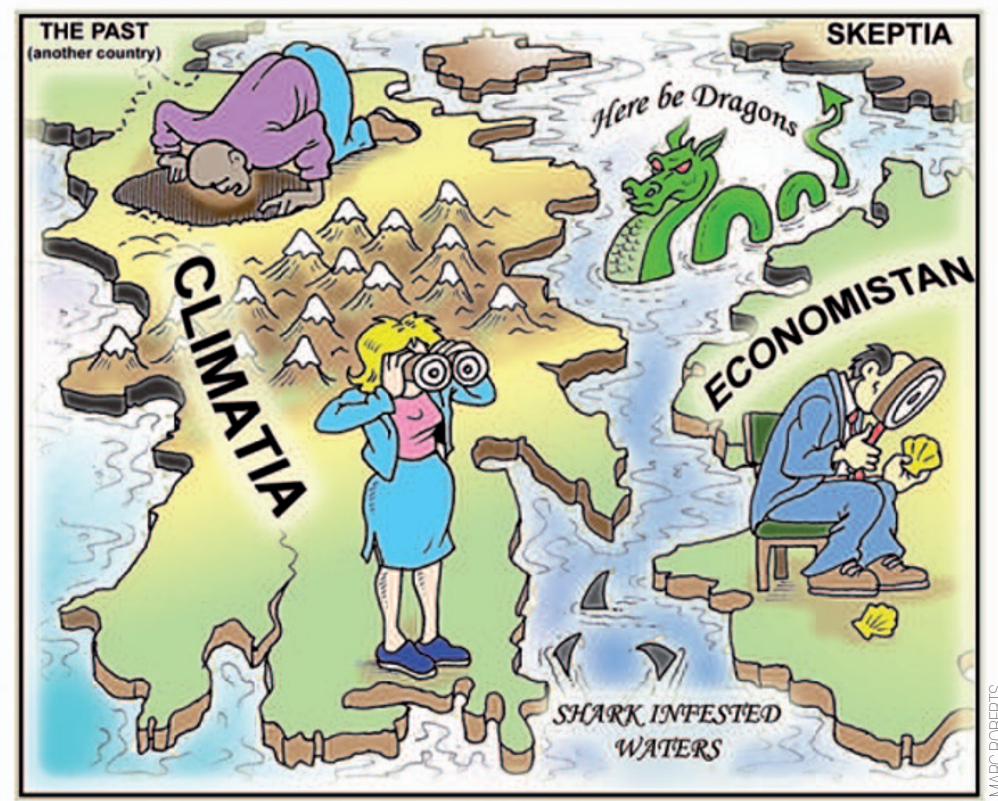

to find proxies for climate change. They must work with data that is erratically sampled and temporally sparse, and whose relation to the underlying physical processes is often mediated by complex biology and geochemistry. Climate modellers often do not appreciate either the difficulties or the successes in the field and may assume that palaeo-science is too anecdotal, qualitative and localized to be of use for quantitative modelling. Palaeoclimatologists, in turn, may suspect that models cannot simulate the large climate changes in the past and assume that modellers have a myopic view of climate history. Given that palaeo-data often uses depth (in an ice or ocean core) as an analogue for time, the difference, colloquially stated, is that modellers focus on the horizontal while Palaeoclimatologists focus on the vertical.

The gap between economics and climate science is perhaps more profound and to a large extent relates to each field's approach to complexity. Over the last 40 years, climate models have evolved into large-scale numerical simulations that represent the physical world in intricate detail. Climate scientists have built this complexity into their models because of their confidence in the underlying fundamental physics of radiative transfer and the equations of motion. Confidence in the results of climate models has been boosted by their predictive success over recent decades and that of closely related weather forecasting models.

By contrast, economists have no hard-and-fast laws analogous to physical processes on which to rely. Human behaviour and social processes are exceedingly complex and poorly understood, and impossible to represent with the type of precision and confidence enjoyed by physical scientists. Economists are therefore typically wary of excessive complexity in calculations and models, taking the view that this rarely improves insight. Their goal instead has been to find simple analytical forms that capture fundamental features of behaviour. To a climate scientist, an economic model can seem rudimentary. To 
an economist, a climate model can seem like a Rube Goldberg contraption.

\section{CULTURE CLASH}

These conflicting attitudes matter because the two fields must be combined to produce analyses that can usefully guide society's response to a changing climate. For instance, regional changes in precipitation could have important impacts on agriculture and infrastructure needs. Only by combining detailed modelling of economic responses with spatially and temporally complex climate projections can we appropriately direct resources for mitigation and adaptation. Without the synthesis of these fields, policy responses can be incoherent and counter-productive, as in the case of the recent rush to biofuels.

Much of the difficulty in bringing fields together relates to the institutional arrangements that emerge from the patterns of thinking in each field. Climate modelling is necessarily a large-scale endeavour, with dozens to hundreds of researchers working on different aspects of a single model. Much labour is provided by young researchers who are expected to spend five to seven years in $\mathrm{PhD}$ programmes followed by a postdoctoral position, meaning that the brightest minds cycle through several different institutions and projects and make contributions to each. In economics, on the other hand, projects are mostly small-scale or even solitary: academic success requires that doctoral students write not only first-author but sole-author papers, and top economics graduates then receive faculty job offers immediately. The time available to make a name for oneself is therefore constrained to a short residence at graduate school, not long enough for a large collaborative project to come to fruition, even were such a project rewarded.

Although palaeoclimate researchers may have more time available, interdisciplinary collaboration may be no easier. In palaeoclimatology, the mark of academic success is often the publication of a new record of precise geochemical analyses that is years in the making. Once a faculty job has been secured, startup funds generally provide for more of the same kind of work. Laboratories equipped with technicians and sample-hungry machines commit the researcher to a mode of investigation that can be difficult to get away from - even for a short time.

But some successful efforts have helped bridge these disciplinary gaps. Climate models are beginning to include some of the physics and biology associated with palaeo-proxy indicators - for instance, models can now incorporate the physics of water stable isotopes and compare them to isotope ratios measured directly from ice cores and cave records, rather than relying on indirect and sometimes ambiguous temperature estimates derived from these proxy data. Palaeoclimate data is now compiled and gridded for easier comparison to model simulations of the last few centuries or the last glacial period, 20,000 years ago. And integrated assessment models combine the work of climatologists and economists. In both arenas, though, fully collaborative efforts are rare.

\section{CULTIVATING COLLABORATION}

How can progress be accelerated? The strategy for building up interdisciplinary science must be twofold: developing people who are comfortable in multiple fields - who can think like both Palaeoclimatologists and modellers, or like economists and climatologists - and providing them with the resources they need for continued interdisciplinary work. The goal must be to create both an intellectual and an institutional environment where these kinds of people and projects can flourish. Given the conservatism of university departments, a shift in the academic reward structure towards greater recognition for collaborative work may be a long time coming. Rather than minimizing or removing existing institutional barriers, the most productive approach may well be to start new institutions.

\section{It is not enough to intermittently bring researchers in different fields together; to accelerate progress, we need to cultivate a new kind of scientist, one who will be able to bridge interdisciplinary divides.}

A lesson can perhaps be learned from the development of climate models themselves. Most of the large modelling efforts are based at dedicated research centres with central, directed and stable funding. In contrast, purely university-based modelling groups have proven hard to sustain. The resources and intellectual merit of bespoke centres draw high-quality researchers to permanent or temporary positions. Staff are understood to be working towards a common goal, and papers with dozens of authors are not uncommon. Interaction with many universities is the norm. At minimum, external university researchers participate as enthusiastic analysers of model output made available in public archives. Academic reputations are made through innovative use of the modelling tools or through refinement of specific aspects of the codes, which continually builds on the work of the group as a whole.

Interdisciplinary centres need not follow this exact model. They can be physical or virtual, university-based or independent, permanent or temporary - recurrent summer programmes, for instance. But all must have the aims of making interdisciplinary science a recognized and valued endeavour, providing a stable base of funding and introducing young researchers at the start of their careers to the issues and needs of the field. If centres are to be virtual, they may still host joint graduate programmes, and they would be best supported by external research funds earmarked specifically for collaborative work so that there is no perceived conflict with more traditional approaches. Several governmental agencies have already begun to try dedicated interdisciplinary funds. For example, the Natural Environment Research Council in the United Kingdom sponsors the RAPID program, which has brought together palaeoceanographers, observationalists and modellers to study changes in the North Atlantic Ocean circulation, and the National Science Foundation in the United States sponsors the Polar Synthesis initiative, which funds only projects that explicitly include distinct fields. In both cases, working across fields is mandated and a significant portion of the resources go to developing student and post-doctoral talent. After slow starts, coherent interdisciplinary communication has been enhanced.

However, junior researchers coming out of these programmes must be assured that an interdisciplinary career is possible. There is little point in training scientists for positions or funding streams that do not exist. Long-term programmes are needed. Here, private foundations can also play an important role in promoting innovation and lending these programmes the prestige and independence required to draw the brightest researchers. The question of how to promote interdisciplinary science is not an abstract one. Without large-scale collaborative work, we may fail to make progress on this defining issue of the twenty-first century. That indeed would be a tragedy.

Published online: 31 July 2008

doi:10.1038/climate.2008.76

Gavin Schmidt is a climate modeller at the NASA Goddard Institute of Space Studies, New York, and is a founding contributor of the Real Climate blog. Elisabeth Moyer is an atmospheric scientist in the Department of the Geophysical Sciences, University of Chicago. e-mail: gschmidt@giss.nasa.gov 